\title{
Actions environnementales et élimination du Trachome dans les zones d'intervention du Programme National de Lutte Contre la Cécité (PNLC) dans la région de Koulikoro
}

\section{Environment actions and elimination of trachoma in the intervention areas of the National Program for the Control of Blindness (PNLC) in the Koulikoro region}

\author{
Kéïta ${ }^{1}$ NF., Touré2 ${ }^{2}$., Dembélée ${ }^{3}$ M., Bamani ${ }^{3}$ S., Diop ${ }^{4}$ S. ; Diakité2 ${ }^{2}$., Sangho ${ }^{4}$ O., Traorée MM., Sangho ${ }^{4}$ A. Doumbia ${ }^{4}$ L.
}

\begin{abstract}
1 Faculté de Médecine; 2 Institut National de Santé Publique; 3 Programme National de Lutte Contre la Cécité; 4 Département d'Enseignement et de Recherche en Santé Publique, ${ }^{5}$ Faculté de Pharmacie, USTTB
\end{abstract}

Auteur correspondant : oussou_toure@hotmail.com

\section{RESUME}

L'objectif de la présente recherche était d'apprécier l'association entre les progrès réalisés en termes d'amélioration des conditions d'hygiène individuelle et environnementale et la baisse de la prévalence du trachome, dans le district sanitaire de Koulikoro en 2013. Cette étude transversale a porté sur un échantillon tiré des populations d'enfants de 1 à 9 ans dans le district sanitaire de Koulikoro. Les données collectées par interview et observation ont été nettoyées, traitées et l'analysées par les logiciels Excel, Accès Microsoft (MAD) et EPIINFO 2000. Le Test de Fischer a été utilisé pour vérifier l'association entre les variables pour un seuil de signification de $p=0,05$. Sur un total de 3653 enfants de 1-9 ans enquêtés, la prévalence du trachome était de $0,8 \%$ chez ceux ayant un visage propre contre $2,4 \%$ chez ceux ayant un visage sale $(p=0,005)$. Dans les ménages disposant de latrines, la prévalence du trachome était de $0,4 \%$ chez les enfants contre 3,6\% chez ceux des ménages sans latrines $\left(p=10^{-8}\right)$. Elle était de $0,4 \%$ chez les enfants des ménages utilisant les latrines contre $5,3 \%$ chez les enfants vivant dans les ménages n'utilisant pas de latrines $\left(p=10^{-8}\right)$. En terme de source d'eau potable, la prévalence du trachome chez les enfants vivant dans les concessions qui ont une source d'approvisionnement était de $0,3 \%$ contre $0,5 \%$ chez les enfants vivant dans les ménages ayant leur source d'eau hors du village $(p=0,005)$. Le nettoyage du visage, l'approvisionnement en eau, l'utilisation des latrines ont contribué à la diminution de et la prévalence du trachome actif chez les patients de 1 à 9 ans.

Mots clés: Trachome, prévalence, chance, Koulikoro, Mali.

\footnotetext{
ABSTRACT

The objective of this research was to assess the association between the progress made in terms of individual and environmental hygiene conditions' improvement and Trachoma prevalence in the health district of Koulikoro in 2013. This cross-sectional study was conducted on a sample drown from a population of children aged 1 to 9 . Data collected by interviews and observations were filtered, cleaned and analysed using
}

Excel, Access and Epi info. Fisher Test has been used to appreciate the association between variables for a significance level of $p=0.05$. Of 3653 children aged 1 to 9 surveyed, the prevalence of trachoma was $0.8 \%$ among those who had clean face compared to $2.4 \%$ for those who had a dirty face $(p=0,005)$. In children living in households that have latrine, the prevalence of trachoma was $0.3 \%$ compared to $3.6 \%$ in those living in households that do not have latrine $\left(p=10^{-8}\right)$. The prevalence was $0.4 \%$ in children that utilize latrine versus $5.3 \%$ in children that do not utilize latrine $\left(p=10^{-8}\right)$. In terms of the availability of potable water, trachoma prevalence was $0.3 \%$ in children that have a source of water in their house while those that get water outside the village had a prevalence of $0.5 \% \quad(p=0,005)$. Face cleanliness, drinking water supply, and latrine utilization contributed to active Trachoma prevalence reduction among children aged 1 to 9 .

Key words: Trachoma, prevalence, chance, Koulikoro, Mali.

\section{INTRODUCTION}

La stratégie globale de l'OMS appelée (CHANCE) [1,2] se décompose en quatre composantes dont deux relèvent de l'hygiène : individuelle (Nettoyage du visage« $N$ ») et collective (Chhangement de l'Environnement« $\mathrm{CE}$ »). Les deux autres sont la $\underline{\mathrm{CH}}$ irurgie « $\mathrm{CH}$ » pour prévenir la cécité chez ceux qui souffrent du trichiasis/d'entropion et l'Antibiotiques « $A$ » (pommade de tétracycline ou azithromycine) pour combattre une infection active à Chlamydia [1,2]. Une gamme d'interventions est possible dans le cadre de ces deux composantes dans les divers programmes de lutte contre le trachome. Les interventions qui portent sur les volets « $N$ » et « $C E$ » doivent être mises en place lorsque la prévalence du TF (inflammation trachomateuse folliculaire) est supérieure à $5 \%$ chez les enfants âgés de 1 à 9 ans [1,2]. Lorsque la prévalence du TF est supérieure à $10 \%$ dans un district, ces interventions doivent s'accompagner d'une distribution d'antibiotiques dans l'ensemble de ce district. De plus, des interventions chirurgicales de masse doivent être envisagées dès lors que la prévalence du TT dans un district est supérieure à $1 \%$. Les activités de promotion de l'hygiène pour lutter contre le trachome ont pour objectif d'augmenter la proportion des enfants qui ont des visages toujours propres. II a été démontré que la promotion de l'hygiène peut réduire le trachome actif. [1,2]. L'inclusion de la 
promotion de l'hygiène dans un programme de lutte contre le trachome commence dès le début de la mise en œuvre de la stratégie CHANCE.

Les mouches synanthropiques, et en particulier Musca sorbens, sont reconnues comme vecteur potentiel du trachome puisqu'elles peuvent transporter Chlamydia trachomatis et la déposer sur l'œil d'un enfant sain [3]. Des études ont clairement démontré que la présence de mouches sur le visage des enfants, était associée à un risque accru de trachome; d'où l'importance du contrôle des mouches pour diminuer la fréquence du trachome. Par ailleurs, de nombreuses espèces de mouches peuvent transporter du matériel fécal après y avoir déposé leurs œufs et être ainsi vectrices d'agents pathogènes présents dans les excrétas animaux ou humains [4].

S'attaquer aux mouches adultes par épandage extensif d'insecticides risque d'avoir peu d'effets sur leur densité et parait actuellement inenvisageable. La meilleure approche repose donc sur l'assainissement et la suppression des sites de ponte potentiels : Eviter la défécation à l'air libre, les latrines non couvertes, et les dépôts d'ordures anarchiques. En effet, le cycle d'une mouche domestique commune comme Musca domestica varie entre 10 et 14 jours, l'œuf déposé se transformant au bout de 6 à 7 jours en une larve qui migrera dans le sol et éclora ensuite. II est donc important d'éliminer ordures et excrétas avant ce délai, Musca sorbens pondant ses œufs préférentiellement sur les excrétas humains déposés au sol et non pas dans les latrines [5]. Les campagnes d'information-éducation-communication promouvant les pratiques d'hygiène avec une quantité d'eau qui peut être faible en cas de rareté auront d'autant plus de succès qu'elles s'inscriront dans un programme plus vaste d'amélioration de l'approvisionnement en eau qui les rendra alors crédibles. II faut rappeler qu'en France, il a fallu que les conditions de confort progressent et que l'eau soit moins rare pour que le bain quotidien des nourrissons se généralise à la fin du XIXe siècle [6].

CAIRNCROSS (1987) a observé dans les villages du Mozambique que la réduction de la distance à l'eau, et en conséquence du temps passé à la chercher s'accompagnait d'un accroissement notable de la consommation et que cet accroissement correspondait pour $70 \%$ au bain et au lavage des vêtements [7]. La part de l'eau utilisée pour les enfants passait de $1 \%$ à 10 $\%$ du total consommé.

Laver le visage des enfants plusieurs fois par jour est l'une des mesures de la stratégie "CHANCE » et a fait la preuve de son efficacité. [1,2] II faut bien sûr que la quantité d'eau soit suffisante, mais surtout que cela devienne une habitude pour la mère. La diffusion de ces pratiques d'hygiène passe par des campagnes " d'information, éducation et de communication ". La stratégie CHANCE est maintenant bien engagée dans (12) douze pays concernés par le trachome. Une évaluation a été menée entre 2002 et 2004 par KUPER et al (2005) dans (8) huit pays : l'Ethiopie, le Ghana, le Mali, le Maroc, le Népal, le Niger, la Tanzanie et le Vietnam. La mise en œuvre apparaît très inégale selon les Etats [9]

Bien que la pierre angulaire du traitement du trachome reste l'antibiothérapie, il a été démontré que l'amélioration du niveau d'hygiène permet aussi une réduction de la prévalence du trachome même en l'absence de toute modification de la situation économique. De telles expériences ont amené l'OMS à recommander la stratégie "CHANCE ». Du fait de leur nature, ces actions nécessitent non seulement une véritable coopération entre les différents services de santé primaire mais aussi une participation active et prolongée de la part des populations concernées. [10] La présente étude a évalué le lien entre la mise en œuvre des actions de nettoyage du visage des enfants de 1 à 9 ans, le changement de l'environnement (utilisation des latrines par tous les membres des ménages et la disponibilité de l'eau potable), et la prévalence du Trachome.

\section{MATERIEL ET METHODES}

Type et période d'étude :

II s'agissait d'une étude transversale qui a été conduite entre le 26 Mai et le 14 Juin 2013 dans le district sanitaire de Koulikoro qui comptait 19 aires de santé dont 17 étaient dotées CSCom fonctionnel répartis entre une commune urbaine et 8 communes rurales. L'enquête portait principalement sur les enfants de 1 à 9 ans et secondairement les adultes de 14 ans et plus.

\section{Echantillonnage}

L'échantillonnage a été fait en deux étapes : La sélection des grappes et celle des ménages

\section{1 ère étape d'échantillonnage}

Le district était divisé en plusieurs sous-ensembles appelés sous-districts. Chaque sous-district regroupait plusieurs aires de santé contiguës totalisant une population environ 50000 habitants.

Les grappes étaient sélectionnées en utilisant la technique de la probabilité proportionnelle à la population (PPP). Une liste exhaustive des villages du sous district a été établie avec les populations actualisées et cumulées. Dix-sept grappes ont été choisies pour chaque sousdistrict soient 68 grappes pour les quatre sous-districts. L'intervalle d'échantillonnage était obtenu en divisant la population cumulée du sous-district par 17 (17 étant le nombre total de grappes à obtenir par sous-district). Un nombre au hasard entre 1 et l'intervalle d'échantillonnage était choisi de façon aléatoire pour sélectionner la première grappe. Ensuite les 16 autres grappes ont été choisies en ajoutant successivement l'intervalle d'échantillonnage.

\section{2ème étape d'échantillonnage}

La deuxième étape concernait une sélection au hasard de 30 ménages dans chaque grappe. Pour les objectifs de l'enquête, un ménage était défini comme: (i) Un homme, sa femme ou ses femmes plus tous les 
dépendants, (ii) Une veuve plus ses dépendants, (iii) Un grand frère ou sœur et ses dépendants s'ils sont orphelins. Une modification de la procédure décrite par Turner et al. était utilisée pour l'échantillonnage des ménages une fois que les grappes étaient choisies.

Quand l'équipe arrivait au village, elle rencontrait le chef de village, ses conseillers et les relais communautaires, leur expliquait le but de l'enquête en vue d'obtenir leur accord. Si cet accord était obtenu, elle dressait avec eux la liste exhaustive des ménages du village tout en faisant des segments de 5 ménages. Le dernier segment pouvait être constitué de quatre ménages ou de six ménages

Chaque segment était numéroté sur un bout de papier et mis dans un récipient comme une tasse ou un chapeau. Les bouts de papier étaient suffisamment mélangés avant le tirage aléatoire de 6 numéros de segments. Le tirage se faisait par les représentants du village. Si le nombre de ménages du village était inférieur ou égal à 30 , tous les ménages dans le village étaient examinés. Les ménages choisis n'étaient pas remplacés quand les résidents étaient absents ou refusaient un examen. Pour minimiser le nombre de résidents manquants dans les ménages choisis, des équipes d'enquête revisitaient le ménage avant de quitter le village le jour de l'enquête. Les enfants de moins de 6 mois n'étaient pas soumis à l'examen

\section{Critères d'inclusion et de non inclusion}

Ont été inclus tous les enfants âgés de 1 à 9 ans et tous les adultes de 14 ans et plus : (i) résidant dans les villages sélectionnés du district sanitaire de Koulikoro, (ii) appartenant aux ménages sélectionnés, et (iii) qui ont donné leur accord ou assentiment de participer à l'enquête.

N'ont pas été incluses (i) les personnes habitant dans les villages non sélectionnés du district sanitaire de Koulikoro et les personnes qui n'ont pas donné leur accord, et (ii) les personnes n'appartenant pas aux ménages sélectionnés.

\section{Choix des examinateurs et des enquêteurs}

Des examinateurs expérimentés chargés de conduire les équipes d'enquête sur le terrain ont été formés pendant deux jours. La formation portait sur la manière de choisir les ménages dans une grappe sur les formulaires de collecte de données (Tablette ou fiche d'enquête) et aussi la classification simplifiée du trachome selon l'OMS. Cette formation était sanctionnée par un test de validation.

Des enquêteurs ont été aussi formés : (i) à l'utilisation de la tablette avec le logiciel "SIWIFTINSIGHT », et (ii) au remplissage des fiches d'enquête. Ils devaient également valider leur formation.

\section{Travail sur le terrain}

Chaque individu était examiné (yeux) à l'aide d'un casque loupe avec un grossissement 2.5 en vue de dépister les signes de trachome. L'œil droit était examiné en premier ensuite l'œil gauche. La présence d'écoulement oculaire ou nasal était observée chez les enfants de 1 à 9 ans. Pour chaque individu examiné l'enquêteur demandait le nombre de prise de l'azithromycine. La question sur la scolarisation concernait seulement les enfants de 5 à 15 ans.

Un adulte du ménage était interrogé pour déterminer : (i) la présence et l'utilisation de la latrine, et (ii) la source d'eau primaire.

Le contrôle de la qualité

Après chaque ménage l'équipe revisitait les données recueillies avant de passer au suivant. Une seconde visite était organisée à la recherche des absents. Un superviseur vérifiait et sauvegardait les données collectées dans les formulaires.

Traitement, analyse et Interprétation des données

Les données recueillies étaient nettoyées à partir du logiciel Excel, traitées avec Accès Microsoft (MAD) et analysées avec un logiciel statistique avancé EPI Info. Les variables incluaient la résidence (le ménage, la grappe, le sous-district), le sexe, l'âge, le consentement, propreté du visage (l'écoulement oculaire et nasal si âgé de 1 à 9), la scolarisation (si âgé de 5 à 15), stade du trachome, la présence ou l'absence de latrine, et la source d'eau primaire. Les résultats d'enquête étaient exploités pour toutes les activités de lutte contre le trachome. L'interprétation des résultats était en fonction du degré de signification $(p=0,05)$ était choisi comme le seuil de jugement. Selon le tableau de Fischer et de Chi2 si la valeur de $p>0,05$, on concluait qu'il n'y a pas de lien entre les variables qualitatives. Cependant si $p<0,05$, alors il y avait un lien entre les variables.

\section{Considérations éthiques}

Les autorités, les chefs de village et les chefs de ménages concernés ont donné leur consentement éclairé. Les individus ont adhéré de façon volontaire à l'enquête et gratuitement. Les cas de trachome actif recevaient gratuitement un tube de pommade tétracycline. Les cas de trichiasis étaient orientés vers les unités de soins au niveau d'un district sanitaire pour une prise en charge gratuite.

Les données recueillies ne servaient pas à d'autres fins et restaient confidentielles.

\section{RESULTATS}

Sur un total de 3653 enfants de 1 à 9 ans vus, le sexe féminin représentait plus de la moitié avec $53,80 \%$ des patients. La tranche d'âge des patients de 5-9 ans représentait plus de la moitié avec $56,30 \%$ contre $43,70 \%$ pour les $1-4$ ans. Chez les patients de 14 ans et plus, sur 4532 patients enquêtés, $44,20 \%$ étaient de sexe masculin et $55,80 \%$ de sexe féminin soit un sex ratio M/F de 0,79 ; ainsi, dans les deux tranches le sex ratio était en faveur des femmes.

La prévalence du trachome actif était de 0,50\% (Tableau I), dont $0,9 \%$ chez les enfants de sexe féminin, et $0,2 \%$ chez ceux du sexe masculin (Tableau II). II existe un lien très significatif entre le trachome actif et le sexe $(p=$ 0,004). 
La prévalence du trachome actif était pareil quelle que soit la tranche d'âge, soit $0,50 \%(p=0,88)$ (Tableau II). La proportion des patients de 1 à 9 ans qui avaient le visage propre était de 22,9\% (Tableau IV). Parmi les enfants de 1-9 ans enquêtés, la prévalence du Trachome était de $0,8 \%$ pour les patients ayant un visage propre ; celle de ceux ayant un visage sale était $2,4 \%(p=0,005)$ (Tableau V). II existait un lien très significatif entre le nettoyage du visage et la prévalence du trachome actif chez les patients de 1 à 9 ans.

La prévalence du Trachome était de 0,3\% chez ceux vivant dans les ménages disposant de latrines; elle était de $3,6 \%$ dans les ménages sans latrines (Tableau II), $\left(p=10^{-8}\right)$, d'où l'existence de lien très significatif entre l'existence des latrines et la prévalence du trachome actif. Cette prévalence du Trachome était de 5,3\% chez les enfants vivant dans les ménages n'utilisant pas de latrines alors qu'elle était seulement de 0,4\% dans les ménages utilisant les latrines (Tableau II), $\quad\left(p=10^{-8}\right)$. II existe un lien significatif entre l'utilisation des latrines et la prévalence du trachome actif chez les enfants 1 à 9 ans.

Seulement $14,94 \%$ des ménages avaient une source d'eau dans la concession et $83,41 \%$ s'approvisionnaient à une source située dans le village. La prévalence du trachome actif était de $0,5 \%$ chez les enfants vivant dans les ménages qui avaient leurs sources d'eau hors du village alors qu'elle était seulement de 0,3\% pour ceux qui s'approvisionnaient en eau dans la concession (Tableau II), ( $p=0,005)$. II existe un lien significatif entre l'approvisionnement en eau et la prévalence du trachome actif chez les enfants de 1 à 9 ans.

\section{DISCUSSION}

Chez les patients de 1 à 9 ans, sur 3653 patients enquêtés, le sex ratio M/F était de 0,85 . Chez les patients de 14 ans et plus, sur 4532 patients enquêtés, le sex ratio $M / F$ était de 0,79 . Aussi, dans les deux tranches le sex ratio était en faveur des femmes. Cette prédominance féminine reflète l'état même de la population du Mali en général et particulièrement celle de Koulikoro. Par ailleurs, les femmes étaient les plus présentes à la maison lors de l'enquête.

Parmi les enfants de 1 à 9 ans, la tranche d'âge la plus représentée était celle des enfants de 5-9 ans.

En 2009, le taux de prévalence du trachome était de 8,7\% [7,5-9,9]. En 2013, après 3 ans (2009-2012) de mise en œuvre de la stratégie CHANCE, le taux de prévalence de trachome actif était retombé à 0,5\% [0.3$0.8]$. Les patients de 1 à 9 ans de sexe féminin avaient le taux de prévalence le plus élevé soit 0,90\% TF/TI. Ceci s'expliquerait par le fait que le sexe féminin, même en bas âge, est plus exposé au trachome que le sexe masculin, du fait d'être en contact constant avec d'autres enfants.

La tranche d'âge 5-9 ans avait le taux de prévalence le plus élevé soit $0,5 \%$. Ceci pourrait s'expliquer par le fait qu'à cet âge, les enfants, encore très jeunes, reçoivent moins d'attention de la part des parents qui s'occupent plutôt des plus petits.

Une étude réalisée à Dioïla par LONGTCHIS S. en 2005 a montré que la prévalence du trachome actif est passée de $33,50 \%$ en (1996-1997) à $0,81 \%$ en 2005 chez les enfants de moins de 10 ans [11].

Bien que des efforts aient été fournis pour lutter contre le trachome, sa prévalence reste élevée dans le district sanitaire de Koulikoro. Elle reste au dessus du seuil d'élimination de l'OMS qui est de 0,1\%.

En effet, dans la présente étude, sur 3653 patients de 1 à 9 ans enquêtés, $22,90 \%$ avaient le visage propre pour une prévalence du trachome actif de $0,8 \%$ alors qu'en 2009 la prévalence du Trachome était de $0,3 \%$ pour une proportion d'enfants à visage propre qui s'élevait à $53,10 \%$. La proportion des enfants à visage propre reste inferieure non seulement à celle de 2009 mais à celle recommandée par l'OMS (plus de $80 \%$ ). Cette proportion était aussi de loin inférieure à celle de Fana avec $86 \%$ en 2009 [14]. Une étude réalisée par Germain M. en 2000 au Mali a montré que la prévalence du trachome actif était de $18 \%$ pour ceux qui avaient un visage propre et $58 \%$ pour ceux qui avaient un visage sale [15]. Le rôle de la réduction du trachome actif par le nettoyage du visage avait été déjà démontré par Resnikoff .S et Queguiner [16]. Francis $V$ et Turner $V$ avaient montré que le visage propre attirait moins de mouches et réduisait le risque de se faire infecter [17]. II existe des études qui ont prouvé que se laver le visage plusieurs fois par jour permet de diminuer la prévalence du trachome actif [18]. En conséquence, cette augmentation de la prévalence serait imputable à un arrêt ou à une diminution des campagnes de sensibilisation ou d'éducation à l'hygiène notamment le lavage du visage.

Sur les 2169 ménages enquêtés, 2134 ménages disposaient de latrines soit $98,40 \%$. Dans $98,30 \%$ de ces ménages disposant de latrines, ces dernières étaient utilisées par tous les membres, et la prévalence du trachome actif était de 0,3\% chez les enfants de 1 à 9 ans. En 2009, le taux d'utilisation de latrines était de 99,6 $\%$. Ces résultats indiquent aussi une baisse de l'utilisation des latrines.

En 2013, une étude réalisée par Haile $M$ et al. en Ethiopie sur un échantillon aléatoire d'enfants âgés de 0 à 9 ans suivi longitudinalement a montré que pour chaque augmentation de $10 \%$ dans l'utilisation des latrines, il y avait une diminution de 2,0\% [0,2-3,9] de la prévalence de la communauté de chlamydia oculaire sur l'année suivante $(p=0,04)$ [19]. La promotion des latrines et de leur usage par tous, même par les enfants diminuerait la prévalence du trachome actif [20].

Sur un total de 3653 enfants de 1 à 9 ans enquêtés, seulement $17,20 \%$ s'approvisionnaient en eau dans la concession et $81,10 \%$ s'approvisionnaient dans le village (environ à $900 \mathrm{~m}$ de la concession) pour des prévalences respectives du trachome actif de $0,3 \%$ et $0,4 \%$ chez les enfants de 1 à 9 ans. 
En 2009 ces proportions étaient respectivement $13,7 \%$ et $42,2 \%$. Ceci confirme l'impact de la disponibilité de l'eau sur le statut du trachome actif ; plus l'eau est disponible et utilisée pour l'hygiène moins la prévalence du trachome est élevée. Une enquête réalisée au Mali par Scheman JF. et al. a montré que la prévalence du trachome augmentait en fonction de l'éloignement par rapport à une source d'eau [21].

\section{CONCLUSION}

La présente étude a confirmé l'impact positif de la composante environnementale de la stratégie "CHANCE ». En effet, il apparait un lien positif entre la propreté du visage, l'utilisation des latrines par tous dans les ménages et la disponibilité de l'eau potable, et la réduction de la prévalence du Trachome. Malheureusement, les indicateurs de la composante environnementale de la stratégie "CHANCE" ont pu progresser ; dans certains cas, ils ont même régressé dans la zone d'intervention du Programme National de Lutte Contre la Cécité, entre 2009 année de démarrage du projet et 2013 année de la présente étude. II apparait ainsi que les volets Nettoyage du visage et Changement de l'Environnement, n'ont pas bénéficié de l'attention qu'ils méritent dans la mise en œuvre du projet. En conséquence les résultats globaux n'ont pas atteint les niveaux escomptés. Aussi, une attention particulière doit être portée à cette composante pour conforter les résultats positifs enregistrés, afin de tendre vers une élimination du Trachome dans le District de Koulikoro et répliquer l'expérience dans le reste du pays.

\section{REMERCIEMENTS}

Toute notre gratitude au coordinateur du PNLC et ses collaborateurs pour l'appui technique financier et technique ayant permis l'aboutissement de la présente étude. Nos remerciements aux responsables des services de santé du District de Koulikoro, aux autorités locales et traditionnelles des communes et des villages, ainsi que les chefs des ménages dont la coopération a été indispensable.

\section{REFERENCES}

1- West S, Munoz B, Lynch M, Kayongoya A, et al.; Impact of face washing on trachoma in Kongwa, Tanzania. Lancet.1995; 345 : 155-8.

2- Taylor HR, West S, Mmbaga BBO, et al.; Hygiene factors and increased risk of trachoma in central Tanzania. Arch Ophthalmol .1989; $107: 1821-5$

3- Emerson PM, Bailey RL, Mahdi OS, Walraven GE, Lindsay SW.; Transmission ecology of the fly Musca sorbens, a putative vector of trachoma. Trans.R. Soc. Trop. Med. Hyg . 2000; 94 : 28-32.

4- Cairncross S, Feachem R.; Environmental health engineering in the tropics, an introductory text. Second edition. Chichester, John Wiley. 1993: 306p
5- Emerson PM, Simms VM, Makalo P, Bailey RL.; Household pit latrines as a potential source of the fly Musca sorbens -A one year longitudinal study from The Gambia.Trop. Med. Int. Heath. 2005; 10: 706709.

6- Rollet C, Morel MC. ; " Façonner le corps : hygiène, massage et vêtements ». In : Des bébés et des hommes : traditions et modernités des soins aux touts petits, Paris. Albin Michel, coll. La cause des bébés. 2000 : 384p.

7- Cairncross S, Cliff JL.; Water use and health in Mueda, Mozambique. Trans. R. Soc. Trop. Med. Hyg. 1987; 81:51-54.

8- Vetch J. ; An account of the Ophthalmia which has appeared in England since the return of the British Army from Egypt, 1807. London, C. Stower for Longman, Hurst, Rees and Orme. 10p.

9- Kuper $\mathrm{H}$, et al.; Participatory evaluations of trachoma control programmes in eight countries. Trop.Med. Int. Health. 2005 ; 10 :764-772.

10- Organisation mondiale de la sante; La lutte contre le trachome : Perspectives. Who document (PBL) 96.56 Genève, 1996: 1-47.

11- Longchi S, Prisca B. ; Impact de la mise en oeuvre de la stratégie CHANCE dans la lutte contre le trachome dans le district sanitaire de Dioïla en 2005. Thèse Med, Bamako, 2005. 87p. $n^{\circ} 224$

12- Courtright $P$, Sheppard J, Lane S, Sadek A, Schachter J, Dawson CR.; Latrine ownerchip as a protective factor in inflammatory trachoma in egypt.Br J ophtalmol. 1991; $75: 322-5$.

13- Scheman JF, Sacko D, Malvy D, Momo G, Traore L.; Risk factor for trachoma in Mali. International journal of epidemiology. 2002; 31:194-201

14- PNLC (Programme national de lutte contre la cécité). Enquête de prévalence du trachome dans les districts sanitaires (Nioro, Diéma et Fana) après la mise en œuvre de la stratégie CHANCE en Février 2008. 18p

15- Zefack M , Germain IL. Facteur de risque du trachome au Mali. Thèse Médecine, Bamako, 2000. 88p n54

16- Resnikoff S, Queguiner. Trachome .Encyl Med Chir, Ophtalmologie, Elsevier, Paris, 8.037G-10, 21-140 A10, 2000.

17- Francis $V$, Turner $V$. L'appui communautaire en faveur de la lutte contre le trachome. Guide de l'action sanitaire de district. OMS, Genève. 1995. 31p

18- Henry Ejere OD, Mahmoud Babanini Alhassan, Mansur Rabiu. La promotion de la toilette du visage pour prévenir le trachome actif. Cochrane database of systematic reviews ; 2004 
Tableau I : Répartition des patients de 1 à 9 ans selon le statut du trachome actif (TF/TI)

\begin{tabular}{lcc}
\hline $\begin{array}{l}\text { Trachome } \\
\text { Actif (TF/TI) }\end{array}$ & $\mathbf{n}$ & $\%$ \\
\hline Présent & 19 & 0,50 \\
Absent & 3634 & 99,50 \\
TOTAL & $\mathbf{3 6 5 3}$ & $\mathbf{1 0 0 , 0 0}$ \\
\hline
\end{tabular}

Tableau III : Appréciation de l'aspect du visage chez les patients de 1 à 9 ans

\begin{tabular}{lcc}
\hline Visage & Fréquence & $\%$ \\
\hline Propre & 836 & 22,90 \\
Sale & 2817 & 77,10 \\
TOTAL & 3653 & 100,00 \\
\hline
\end{tabular}

Tableau II : Répartition des caractéristiques en fonction du statut de trachome actif (TF/TI)

\begin{tabular}{|c|c|c|c|c|c|}
\hline \multirow{2}{*}{ Caractéristiques } & 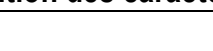 & \multicolumn{2}{|c|}{ Trachome actif (TF/TI) } & \multirow[b]{2}{*}{ Total } & \multirow[b]{2}{*}{$p$} \\
\hline & & Présent & Absent & & \\
\hline \multirow[t]{3}{*}{ Sexe } & & $19(0,5 \%)$ & 3634 & 3653 & \\
\hline & Féminin & $15(0,9 \%)$ & 1672 & 1687 & 0,004 \\
\hline & Masculin & $4(0,2 \%)$ & 1962 & 1966 & \\
\hline \multirow[t]{3}{*}{ Age (année) } & & $19(0,5 \%)$ & 3634 & 3653 & \\
\hline & $1-4$ & $8(0,5 \%)$ & 1589 & 1597 & 0,88 \\
\hline & $5-9$ & $11(0,5 \%)$ & 2045 & 2056 & \\
\hline \multirow[t]{3}{*}{ Latrines } & & $19(0,5 \%)$ & 3634 & 3653 & \\
\hline & Présentes & $10(0,3 \%)$ & 3393 & 3403 & $10^{-8}$ \\
\hline & Absentes & $9(3,6 \%)$ & 241 & 250 & \\
\hline \multirow[t]{3}{*}{ Latrines } & & $19(0,5 \%)$ & 3634 & 3653 & \\
\hline & Utilisées & $15(0,4 \%)$ & 3563 & 3578 & $10^{-8}$ \\
\hline & Non utilisées & $4(5,3 \%)$ & 71 & 75 & \\
\hline \multirow[t]{5}{*}{ Source d'eau } & & $19(0,5 \%)$ & 3634 & 3653 & \\
\hline & Dans la & $2(0,3 \%)$ & 544 & 546 & \\
\hline & concession & & & & \\
\hline & Dans le village & $14(0,4 \%)$ & 3033 & 3047 & 0,005 \\
\hline & Hors du village & $3(0,5 \%)$ & 57 & 60 & \\
\hline
\end{tabular}

Tableau IV : Répartition de la prévalence du trachome actif chez les patients de 1 à 9 ans en fonction l'aspect du visage

\begin{tabular}{llccccc}
\hline & & \multicolumn{2}{c}{ Trachome actif (TF/TI) } & & \\
\cline { 3 - 4 } & & Présent & Absent & & Total & p \\
\hline Aspect du visage & & $74(2,0 \%)$ & 3579 & 3653 & \\
& Sale & $67(2,4 \%)$ & 2750 & 2817 & 0,005 \\
& Propre & $7(0,8 \%)$ & 829 & 836 & \\
\hline
\end{tabular}

